$\mathrm{O}$ ver the last 500 years, there have been, on average, three severe influenza pandemics in each century. The most recent pandemic was declared in 2009. Yet despite much investment in public health and many improvements in vaccine production techniques and know-how, the availability of influenza vaccines during this event was far from adequate. Six months into the pandemic, 534 million doses were available, and after one year that number had risen to 1.3 billion - enough for only $8 \%$ and $25 \%$, respectively, of the world population. We were lucky that the pandemic declared in 2009 turned out later to be mild and that just one shot of vaccine was sufficient to protect most people. This is not usually the case during a severe influenza pandemic.

Unless there are fundamental changes in both influenza vaccine manufacturing and global pandemic preparedness, another pandemic will mean that vaccines will again be in very short supply and available in only a few, developed countries.

\section{LIMITS IN PRODUCTION}

Only a few factors currently limit the timely supply of pandemic vaccines, but unfortunately these factors are fundamental. One is that seasonal and pandemic influenza vaccines are produced by similar technologies in the same facilities, using methods that rely almost exclusively on embryonated chicken eggs. The existing level of production in these seasonal-vaccine facilities can rapidly increase only marginally, and it would take years to bring new facilities online, which means that production cannot be expanded quickly enough to keep pace with the spread of a pandemic virus that will go global within days or weeks of detection.

Even when production is switched to pandemic vaccines, it will take 3 or 4 months for the first doses to become available - by which time outbreaks might have already peaked on some continents. A large part of this delay is the time required to isolate and characterize the virus, prepare the vaccine prototype and adapt the manufacturing process, and prepare reagents for vaccine release.

The production capacity for seasonal influenza vaccines is closely matched to annual demand; it is not sustainable to keep excess manufacturing capacity idle. Over the past ten years, the annual output of seasonal vaccine has doubled to approximately 450 million doses $^{1}$, which can be ramped up in the event of a pandemic to give a total potential capacity of around 850 million doses. However, as was shown during the 2009 pandemic, that is not even close to providing the billions of doses that would be required in the short time available.

On the positive side, there have recently been some promising developments in influenza vaccine research that could address several of these shortcomings. None, however, is yet in later-stage clinical development.
Should we face another pandemic in the next few years, the scope of the vaccine response would probably be similar to that of 2009. There is one major difference, however: over the last few years, a considerable portion of global pandemic production capacity has been pre-booked by some countries. This ensures that they will have access to the precious vaccine doses supplied during the crucial first 6-8 months of a pandemic.

\section{FIVE-YEAR OUTLOOK}

As countries continue to pre-book pandemic supply, it is more and more likely that the limited vaccines available during the first months of any pandemic during the next few years will be sold out almost completely. Some companies might hold capacity to address any manufacturing and vaccine formulation uncertainties. Some might decide to allocate up to $10 \%$ of their pandemic vaccine output to the World Health Organization (WHO), as required in its Pandemic Preparedness Framework Agreement, in exchange for receiving pandemic strains to be used for vaccine production. But these doses will vanish into the large supply gap that will face developing countries.

On the production side, there are no radical changes on the horizon for the next few years. True, some recently introduced vaccines are produced using cell culture and recombinant technology. But most production sites still use eggs - and so, in the near future, the impact of these newer production methods on global supply will probably be limited. The speed with which manufacturers can ramp-up or expand influenza production to meet pandemic demand will not fundamentally change in the next few years.

Vaccine production capacity is driven by demand: the increase in capacity mentioned earlier resulted mainly from improvements in vaccine coverage in developed countries. To put things into perspective, more than three-quarters of the annual seasonal influenza vaccine doses that are produced globally are consumed by just 12 countries in North America and Europe ${ }^{2}$, and most of the rest go to Japan and Australia. Coverage in these countries is showing signs of plateauing. In developing countries, by contrast, the demand for seasonal influenza vaccine is likely to remain negligible in the coming years. In these countries, most immunization efforts will continue to focus on diseases covered by the WHO's Extended Programme on Immunization, together with other diseases such as those caused by rotavirus, pneumococcus and human papillomavirus. With the help of organizations such as the WHO, a few pilot vaccine production plants might go online in Asia, but without sustainable seasonal vaccine demand, large-scale influenza manufacturing in developing countries will remain untenable.

For these reasons, it is highly unlikely that global seasonal 


\section{LATE TO THE PANDEMIC}

Influenza vaccine doses became available in large quantity only after the 2009 pandemic had peaked. Data shown are from a northern hemisphere country that had in place a significant pandemic preparedness program.

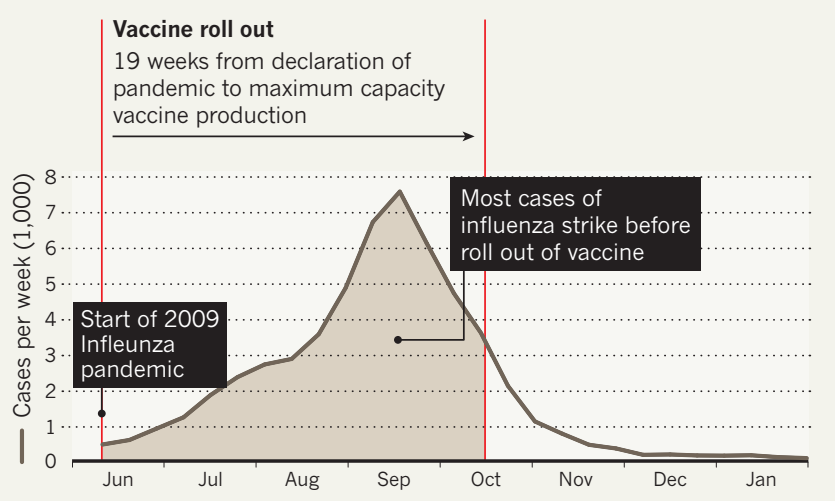

influenza vaccine capacity will increase to the projected 1.75 billion doses in 2015 that was forecast in the WHO's Pandemic Influenza Preparedness Framework. It is much more likely that the maximum annual capacity will remain at around 400 million doses above the seasonal demand of 550 million doses in 2014. As a consequence, instead of capacity increasing, this could lead to current expansion plans not materializing or existing production capacity being shut down in response to market dynamics, including the current rapid price erosion of seasonal influenza vaccines.

One way to stretch the limited seasonal production capacity during a pandemic is to add a proven, safe, antigen-sparing adjuvant, which could multiply vaccine output by as much as 12 times. A safe adjuvant will also be required to increase the duration of protection against the influenza variants that are likely to arise from an initial pandemic virus. Manufacturers of influenza vaccines will probably seek to license the rights to produce an adjuvant that has been developed by another firm, purchase and stockpile a suitable adjuvant or - in what would be a costly and long-term undertaking - develop their own.

Other developments are at hand to improve the speed of vaccine production. Using synthetic biology ${ }^{3}$, it is possible to shorten the time needed to prepare the crucial vaccine candidate seeds from 4-6 weeks using conventional methods to less than 1 week. In 2013, candidate vaccine strains for the H7N9 outbreak were swiftly prepared using such new technologies for the first time ${ }^{4}$. New tests have been developed that expedite the release of vaccine batches, shaving a month off the time needed for vaccine formulation and delivery. These tests are being introduced into the regulatory frameworks so that they can replace the slower existing tests. Together, these advances could mean that the first vaccine doses are ready for use 1-2 months earlier than is possible using conventional methods.

\section{LONG-TERM CHANGES}

Even with a modest increase in production capacity and a small but appreciable reduction in the time taken to deliver the first doses of a vaccine, most of the world's population - especially those outside the developed world - will be as vulnerable as ever to another pandemic in the coming years.

The best hope for a change in the status quo comes from the introduction of revolutionary influenza vaccine production technologies. Ideally, it would be sustainable to run such technologies below capacity, with the possibility of quickly ramping up output in the face of a pandemic. Such technologies, if they also offered economies of scale, could help to prompt the sustainable introduction of seasonal influenza vaccines into developing countries, which would in turn increase demand for the vaccines and thus manufacturing capacity. However, at present the global public health research community seems to be paying little or no attention to this possibility.

The new technologies that seem to have the greatest potential include self-amplifying RNA vaccines and, to a lesser extent, plantderived vaccines and haemagglutinin-based bacterial expression systems. There are several companies investing in RNA vaccines; if this platform and the RNA vaccines prove to be stable, and the encouraging results from preclinical tests are confirmed in humans, this technique could be used to provide almost immediate pandemic vaccine responses. Fill-finishing capacity would need to be externally contracted in advance.

Another potential solution to pandemic readiness would be the development of a universal influenza vaccine that could be either used for pre-pandemic priming or produced at sufficient speed and quantity for use during a pandemic. Either approach would represent a breakthrough in pandemic preparedness; research towards these goals will improve the seasonal vaccine in the interim. But the goal of covering all subtypes of influenza-A with one vaccine, produced in a way that can be ramped up quickly during a pandemic, seems decades away at best.

If the capacity to produce pandemic vaccines quickly is to be increased, it will be important to raise the demand for seasonal influenza doses around the world, including in developing countries. Seasonal influenza has significant health impacts in all climate zones; with sustained economic growth, more countries will be able to afford the vaccines. Such an increase in demand, if sustainable, will lead to the creation of more production capacity in developing countries, and thus to an increase in global capacity.

\section{A NEW APPROACH}

The limitations of the existing influenza vaccine production technologies are the fundamental reasons for the seemingly insurmountable gap in pandemic vaccine supply. Addressing these limitations could help to bring about an affordable and equitable pandemic vaccine supply and also pave the way for the introduction of seasonal influenza vaccines in developing countries. Some of the recently developed vaccine production technologies have the potential to resolve the pandemic vaccine dilemma; they could even have broader applications for the prevention of other communicable diseases. Unfortunately, global public-health authorities have so far failed to make the strategic investments needed to effect these changes in the influenza vaccine landscape. The visible trend seems to be that of maintaining current practice, despite the evidence of its inadequacy during the 2009 pandemic.

National and global health authorities need to change course and embrace these developments. In so doing, they can create a new era of pandemic preparedness.

Klaus Stöhr is vice president and global head of policy at Novartis Vaccines and Diagnostics, Cambridge, Massachusetts, and former head of the WHO Global Influenza Program.

Email:klaus.stohr@novartis.com

1. Collin, N. \& de Radiguès, X. Vaccine 27, 5184-5186 (2009).

2. Palache, A. Vaccine 29, 9459-9466 (2011).

3. Dormitzer, P. R. et al. Sci. Transl. Med. 5, 185ra68 (2013).

4. Settembre, E. C. Hum. Vaccin. Immunother. doi: 10.4161/hv.27600 (2013). 